\title{
La reputación online en el marco de la comunicación corporativa. Una visión sobre la investigación de tendencias y perspectivas profesionales
}

\author{
Alicia Vaquero Collado \\ Villafañe \& Asociados Consultores
}

\section{Palabras clave}

Reputación, online, comunicación corporativa, management, nuevas tecnologías, monitorización, herramientas de comunicación

\section{Resumen}

La convergencia entre la proliferación de las nuevas tecnologías y el auge del management ha favorecido el nacimiento de un nuevo concepto: la reputación online. Esta investigación surge ante la necesidad de conformar un marco teórico, que permita afianzar dicho concepto y profesionalizar su gestión. Con este propósito, se lleva a cabo una recopilación de definiciones y modelos conceptuales procedentes de diferentes disciplinas que configuran el diagnóstico sobre el estado del objeto de estudio. Pese a la perspectiva multidisciplinar de la investigación, la hipótesis principal enmarca la reputación online en el ámbito concreto de la comunicación corporativa. El objetivo es demostrar que la reputación online forma parte de la reputación corporativa y precisa ser gestionada con los mismos indicadores. Así pues, los modelos de reputación online deben alinearse con la estrategia global de reputación de la compañía. Por este motivo, se refutan las concepciones reduccionistas que centran sus disertaciones en la optimización de motores de búsqueda o análisis de las opiniones en redes sociales. El carácter transversal del concepto matriz — la reputación corporativa-, unido a los requerimientos técnicos de la esfera online, motivan el desarrollo de un amplio abanico de roles profesionales. En la investigación se detallan las funciones de dichos profesionales y se analizan las herramientas de gestión y monitorización existentes. El entorno online propicia una nueva forma de relación entre empresas y stakeholders, basada en un proceso comunicativo multidireccional, que favorece la democratización de la conversación y contribuye al desarrollo de la sociedad. 


\title{
Online Reputation in the Framework of Corporate Communication. An Overview on the Research About Professional Trends and Tendencies.
}

\section{Keywords}

Reputation; online; corporate; management; new technologies; monitoring; communication tools

\begin{abstract}
The convergence between the proliferation of new technologies and the rise of management has led to the birth of a new concept: online reputation. This research comes before the need to shape a theoretical framework that strengthens the concept and professionalizes its management. To this end, is being carried out a compilation of definitions and conceptual models from different disciplines that make up the diagnosis on the state of the object of study. Despite the multidisciplinary perspective of the investigation, the leading hypothesis frames online reputation in the specific field of corporate communication. The aim is to show that the reputation online forms part of the corporate reputation and needs to be managed with the same indicators. Thus, the models of online reputation must be aligned with the overall strategy of the company's reputation. For this reason is refuted the reductionist vision that focus their presentations in the search engine optimization and analysis of the views on social networks. The horizontal nature of the concept matrix - corporate reputation-, coupled with the technical requirements of the online field; allow the rise for the development of a wide range of professional roles. In the research appear the details of the functions of these professionals and also some analyses of the existing monitoring and management tools. Online environment leads to a new way of relationship between companies and stakeholders, based on a multidirectional communicative process, which favours the democratisation of the conversation and contributes to the development of the society.
\end{abstract}

\begin{abstract}
Autora
Alicia Vaquero Collado [aliciavaquero1@gmail.com] es consultora de comunicación en Villafañe \& Asociados, donde implementa proyectos de reputación empresarial y liderazgo, así como programas de reputación online. Su trayectoria profesional también está vinculada al mundo académico, como investigadora y formadora en el ámbito de la gestión de intangibles.
\end{abstract}




\section{Introducción: principales temáticas de la investigación}

Durante la última década, el individuo ha cambiado la forma de consumir medios, de comunicarse e incluso de relacionarse con el entorno. La revolución de las TIC ha incidido en, prácticamente, la totalidad de los ámbitos sociales. Algunos expertos, se aventuran a afirmar que estamos inmersos en el inicio de una nueva etapa de la Historia de la Comunicación ${ }^{1}$.

El usuario muestra una conducta social en el uso de la tecnología y la participación colectiva propicia la democratización de la conversación. A su vez, el individuo cuenta con una destacada capacidad de adaptación a los cambios tecnológicos, que desarrolla a un ritmo vertiginoso. Ante dicha situación, las corporaciones han tenido que evolucionar simultáneamente para comprender los nuevos códigos de la era digital (Orihuela, 2011).

Por primera vez, el monopolio comunicacional de las organizaciones se disuelve. El usuario adquiere un gran poder en la esfera online. Empresas y stakeholders dialogan en la Red y queda truncado el esquema de comunicación empresarial unidireccional. Los diferentes públicos ya no sólo se relacionan con las empresas priorizando la escucha sino que inician diálogos multidisciplinares desde sus respectivos roles.

La sociedad de Internet, con el paso del tiempo, se muestra más crítica respecto a las decisiones empresariales y existe una demanda latente de transparencia. Además, crecen las exigencias para alcanzar un desarrollo sostenible de las corporaciones. En definitiva, se proyecta la búsqueda de un equilibrio entre los intereses económicos y sociales.

Este insólito episodio de la historia empresarial ha motivado la especialización de los profesionales de la comunicación, que lejos de controlar los flujos de comunicación, se adentran en un territorio desconocido, inexplorado.

Como resultado de las buenas prácticas empresariales en Internet y el buen comportamiento corporativo en la doctrina del management nace un nuevo concepto: reputación online. De esta manera, se está conformando un marco conceptual que pretende abordar una serie de compromisos adoptados por las empresas y demandados por los stakeholders en el entorno 2.0.

Asimismo, las empresas que operan bajo los criterios de la cultura del corporate, comienzan a integrar una nueva área de actuación que emana como respuesta a las necesidades de la esfera online: la gestión de la reputación online.

No obstante, sobre la reputación online se han construido diversos discursos profesionales, desde varias doctrinas o disciplinas, que en algunos casos no resultan compatibles para establecer una base teórica sólida o aplicar un mo-

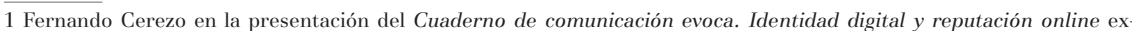
plica que se está construyendo un nuevo paradigma de la comunicación (Cerezo, 2011). Del mismo modo, Jerusalem Hernández alude a una nueva era de la Comunicación (Hernández, 2011). 
delo de gestión eficaz. Resulta necesaria una reflexión acerca de la noción y su potencial futuro.

El objetivo primordial de la investigación es establecer un marco teórico sobre la reputación corporativa online, que permita a académicos y profesionales del sector, trabajar sobre unas bases fundamentadas.

\section{Una aproximación a la reputación online}

En la bibliografía localizada son reducidas las publicaciones académicas y cuantiosos los post que han redactado tanto profesionales como amateurs, quienes se han sentido atraídos por la vorágine de las redes sociales y la gestión de los intangibles.

Por ello, ha sido necesario aplicar un filtro crítico que permitiese trabajar en torno a aquellas hipótesis y objetivos planteados por profesionales o académicos bajo unos criterios de solidez y perdurabilidad, teniendo en cuenta la experiencia y profesionalidad de los autores.

La reputación corporativa, origen del objeto de estudio, ha sellado una primera etapa basada en el asentamiento del concepto. Empresas, instituciones y gobiernos ya incorporan en sus agendas estratégicas la gestión y reconocen su importancia para la creación de valor de la organización (Hannington, 2006: 9).

Es evidente que la reputación online emerge y forma parte de las últimas tendencias en innovación dentro de la gestión empresarial. Se precisa desarrollar capacidades que cubran las expectativas de sus stakeholders, cada vez más informados y con un mayor conocimiento de las nuevas tecnologías. En este sentido, las corporaciones deben llevar a cabo un ejercicio de escucha para aprender a relacionarse con sus diferentes grupos de interés. Si bien la dificultad radica en conseguir que dichas relaciones se consoliden, resultará necesario diseñar una estrategia que contemple las necesidades de cada grupo de interés.

Para ello, el gestor reputacional se concentra en detectar cuáles son las expectativas de los stakeholders hacia la corporación, que van más allá de los cometidos propios de la actividad empresarial que ésta desarrolle.

Además de lo expuesto, cabe añadir que se construyen conjeturas profusas acerca de la afirmación que considera al individuo más severo y crítico en la esfera online que en la offline. Este hecho, alerta a las empresas y éstas, en algunas ocasiones, entablan nuevas experiencias empresariales 2.0 faltas de planificación.

El estudio que se está detallando irrumpió con el objetivo de demostrar la necesidad de integrar totalmente la gestión online con la gestión offline de la reputación de una corporación, mediante la propuesta de metodologías que profundizasen y no se articulasen entorno al mero seguimiento. 


\section{Objetivos e hipótesis de la investigación}

Las premisas sobre las que se cimienta la investigación se han desarrollado de manera lógica y secuencial. La hipótesis inicial emana, en primer lugar, ante la desmesurada cifra de publicaciones relativas a la reputación online, inconexas y desprovistas de un marco conceptual. En segundo lugar, desde la perspectiva del profesional del sector, donde se divisan conatos de gestión reputacional online, carentes de un eje central que permita trabajar bajo criterios comunes y coherentes respecto a la propia reputación corporativa.

Así pues, la hipótesis evidencia que la reputación online forma parte de la reputación corporativa y, por tanto, debe gestionarse bajo los mismos indicadores, teniendo en cuenta, principalmente, los valores corporativos y la jerarquización de stakeholders. Se trata de un concepto innovador en el management actual y precisa un rol profesional que se caracterice por su profundo conocimiento tanto en el ámbito de la comunicación como en el de las nuevas tecnologías.

A continuación se presentan, de forma genérica, los objetivos de la investigación:

- Obtener un compendio de definiciones que justifiquen el área en la que debe ubicarse la reputación online.

- Trazar la evolución del concepto.

- Conocer en detalle el perfil del gestor de reputación online.

- Ubicar el nuevo rol profesional en el organigrama empresarial.

- Evaluar el impacto de la revolución digital sobre la relación empresa-stakeholders.

- Presentar los principales modelos de gestión de la reputación online.

- Analizar las herramientas de gestión y monitorización más empleadas.

\section{Metodología de la investigación}

Es evidente que un fenómeno tan reciente e innovador como el que analizamos, no puede ser abordado de una única manera. Es importante ser ambiciosos, en este sentido, y no renunciar a poder alcanzar una visión amplia y más general. Por ello, a continuación se describe cómo se ha articulado la metodología general de la investigación que se ha desarrollado hasta el momento - en el trabajo de investigación previo al desarrollo de la tesis doctoral-y, en las últimas líneas del artículo, se especificarán los proyectos futuros que permitirán ampliar el estudio.

En primer lugar, se ha llevado a cabo una fase de construcción del marco contextual y teórico. Todo ello, a través de la labor documental que ha permitido 
delimitar las funciones dentro de la disciplina del corporate y el concepto reputación corporativa, desde sus orígenes hasta la actual gestión del management.

También, se ha descrito la evolución del fenómeno digital, basada en conclusiones de investigaciones anteriores. Por otra parte, se han analizado las principales definiciones del término reputación corporativa online y las propuestas de modelos de gestión localizadas en la literatura especializada.

En segundo lugar, la fase del trabajo de campo, ha contribuido a la profundización del objeto de estudio con una investigación cualitativa y ha aportado un gran volumen información sobre buenas prácticas en la gestión reputacional online. Para ello, se ha seleccionado a expertos en el área de reputación online, pese que en algunos casos no proceden de la disciplina de la reputación, que han introducido una visión complementaria, mediante entrevistas en profundidad.

De esta manera, entre los miembros de la muestra se encuentran Cristina Aced, Neus Arqués, Miguel del Fresno, Francesc Grau, Jerusalem Hernández e Iván Pino, profesionales con una extensa trayectoria profesional en comunicación que conciben la gestión de la reputación como labor intrínseca de su desempeño diario.

\section{La revolución digital}

Hace más de una década, cuatro visionarios detectaron la influencia que podría llegar a tener la sociedad de Internet. Fue en 1999 cuando Rick Levine, Christopher Locke, Doc Searls y David Weinberger escribieron el Cluetrain manifiesto, un documento con 95 hipótesis que versaba sobre el comportamiento de las empresas que operan en un mercado con nuevas conexiones. Supuso una llamada de acción a las compañías.

Las ideas expresadas dentro del manifiesto buscaban examinar el impacto de Internet tanto en los usuarios como en las organizaciones. Dos años después, el estallido de la burbuja tecnológica marcó una etapa crucial para la web y la dimensión online. En aquel instante, diversos expertos concluyeron que las expectativas hacia este fenómeno eran excesivas.

Casi tres años después nació el concepto «Web 2.0», a partir de una sesión de brainstorming en la que participaban O'Reilly y Media Live International. El presidente de esta última y Dale Dougherty, pionero de la web, creyeron que contaba con un futuro prometedor en el que se presentaban sorprendentes aplicaciones. Tim O’Reilly y John Battelle compartieron esta misma opinión y protagonizaron la primera conferencia acerca de la Web 2.0.

A principios del siglo XXI, las tesis del Cluetrain manifiesto recobraron protagonismo, ante el nacimiento de los blogs. Google, el buscador más popular del planeta, impulsaba la notoriedad de aquellas páginas web que cumplieran con los estándares fijados por la industria. Sin embargo, poco tiempo después, trasladó 
determinados blogs hasta las primeras posiciones, mostrando al mundo la corriente de conversaciones humanas. Estas empresas confirmaron la hipótesis del Cluetrain Manifiesto, donde se afirmaba que los mercados son conversaciones.

Según O’Reilly (Cobo Romaní, 2007:37), los principios constitutivos de la Web 2.0 son: la web como plataforma, el aprovechamiento de la inteligencia colectiva, la gestión de la base de datos como competencia básica, el fin del ciclo de las actualizaciones de versiones del software, los modelos de comparación ligera junto a la búsqueda de la simplicidad, el software no limitado a un único dispositivo, y las experiencias enriquecedoras de los usuarios.

Se observa cómo la revolución digital cuestiona el modelo tradicional de emisor/ receptor (Esquinas, 2010) y el sistema de intermediación social, representado por los medios de comunicación. La red permite que los roles de los diferentes agentes sociales se construyan y transformen de forma continua. En este nuevo espacio la autoridad no se da por supuesta, por el contrario, se cuestiona y confirma constantemente (Cerezo, 2011).

Explica José Manuel Velasco en el prólogo del libro Mi Comunidad... ¿Me quiere o no me quiere? que «los cambios más revolucionarios son aquellos que se instalan en nuestras vidas de forma casi natural, sin traumas, y acaban convirtiéndose en nuevos paradigmas» (Fuetterer, 2010:5).

En este sentido, la revolución digital responde a un nuevo esquema, caracterizado por un cambio en los hábitos y un estilo de vida irreversible. Se trata de un modelo insólito, sitúa al individuo en el centro del universo, de un espacio personal y, a su vez, colectivo.

El número de profesionales del campo de la comunicación corporativa que muestra un rechazo inicial hacia las nuevas tecnologías continua siendo elevado pese al auge de la revolución digital (Celaya, 2007: 193). La falta de formación supone un obstáculo para gran parte de los directivos de comunicación. Aquellos argumentos que cobran un mayor peso son: la dificultad para controlar contenidos y la integración entre estrategia de comunicación y contenidos. En cuanto a la principal limitación, cabe señalar que es, según los expertos de la muestra, la falta de asesoramiento en el periodo previo a la inmersión en la esfera online.

\section{La nueva forma de relación entre stakeholders y corporación}

Justo Villafañe (2006:11) destaca la necesidad de mantener un diálogo con los grupos de interés de la compañía. Para ello, resulta imprescindible la creación de un marco estable que potencie las relaciones y la ejecución de acciones con un alto valor relacional.

El ya citado marco se consigue cuando la empresa responsabiliza a los departamentos y las personas concretas de la relación de cada stakeholder. De esta manera, la implementación de acciones con un alto valor reputacional se divisa 
como la consecuencia de una adopción de políticas corporativas específicas para cubrir las demandas de los stakeholders, habiéndose comprometido la empresa previamente a garantizar dicha satisfacción.

Resulta especialmente relevante evaluar el grado de apertura de una corporación hacia las nuevas tecnologías ya que permite determinar el nivel de predisposición para conversar en la red (Cerezo, 2011:15).

Cuando una empresa decide relacionarse con sus stakeholders en Internet, en primer lugar, debe prepararse, mediante una formación especializada, que suponga un impulso para interactuar con sus públicos de forma amigable y coherente respecto a sus principios. Así pues, el diseño de un plan de actuación facilitará el transcurso de las relaciones. Con anterioridad, es conveniente realizar un esfuerzo para entender correctamente las reglas de la conversación que tienen lugar en la Internet.

Según Víctor Gil y Felipe Romero (2008:11-13), existen tres factores clave por los que ha cambiado la relación entre los grupos de interés y la empresa. Teniendo en cuenta que esta última debe ser transparente y honesta porque su público así lo demanda:

1. Generalización de una actitud crítica ante los mensajes de las marcas (en este caso podríamos extrapolarlo a las empresas). El usuario de Internet, en general, adopta una actitud escéptica, desconfiada. La tendencia a pensar en la intrusión, la venta, el engaño, etc.

2. La mejora de las competencias por parte del usuario para juzgar las propuestas es un hecho irrefutable.

3. El uso de las nuevas tecnologías y, en concreto, de Internet ha provisto al usuario de herramientas que le permiten obtener un trato directo con las compañías.

\section{La reputación corporativa en el ámbito online}

Es cuantiosa la cifra de publicaciones en las que la gestión de la reputación online y la gestión de los social media son cometidos que se citan indistintamente. Ante la confusión de términos, se propone consensuar una definición y acotar un área de actuación para cada nomenclatura.

La reputación online tanto en artículos como post se vincula, principalmente, a problemas de SEO (Search Engine Optimizacion), optimización de los medios sociales (Social Media Optimizacion, SMO), gestión de crisis, monitorización y reputación personal. Si bien, puede relacionarse con algunos de estos aspectos, entender de tal modo la reputación online supondría formular una visión excesivamente simplista del concepto. 
En efecto, la literatura que versa sobre el objeto de estudio que nos ocupa, ha contemplado la reputación online desde la constante prevención y eliminación de riesgos reputacionales. De esta manera, se ha obviado una posible gestión de la reputación en Internet con fines proactivos, que contribuyan al fortalecimiento de las relaciones con los diferentes grupos de interés.

Con el fin de adentrarnos en el fenómeno, apelamos a un estudio realizado por Weber Shandwick, con la colaboración de The Economist Intelligence Unit (EIU) denominado Risky Business: Reputations Online. La muestra del sondeo la componían 703 altos directivos de compañías de más de veinte sectores diferentes de Norteamérica, Europa y Asia.

Las conclusiones del mentado estudio nos permiten afirmar que cualquier empleado, sin importar el nivel que ocupe en la organización ha podido contribuir a dañar la reputación de su compañía a través de herramientas online. Del mismo modo, el resto de stakeholders pueden incidir negativamente sobre la organización, expresando opiniones en Internet que, más tarde, es posible que aparezcan en las búsquedas cotidianas de los usuarios y condicionen su opinión.

El desarrollo exponencial de las redes sociales ha puesto sobre aviso a los responsables del corporate, concretamente, a aquellos perfiles profesionales encargados de la reputación corporativa, sobre la incidencia de Internet en el manejo cotidiano de los comentarios vertidos en la Red acerca de sus empresas.

Sin embargo, la banalización entorno a la reputación online ha creado cierta confusión sobre su significado, alejándolo, a su vez, del concepto matriz, reputación. No todas las opiniones ni acciones de los usuarios del medio Internet pueden ser consideradas amenazas reputacionales (Carreras, 2008).

Definir la gestión reputacional online como una cuantificación de la información negativa vertida sobre la empresa, puede fomentar el intrusismo, puesto que esta argumentación se apoya en prácticas poco profesionales y rigurosas. Las herramientas que facilitan la detección y posterior reporte de las opiniones de los usuarios del medio Internet, distan en exceso de la gestión de la reputación online. De nada sirve que durante un determinado periodo de tiempo se generen críticas positivas sobre una organización si ésta no se rige según dictan sus principios. Así pues, las opiniones aisladas de los usuarios, únicamente son juicios de valor sin relevancia en la opinión pública.

En este caso, el principal objetivo se fundamenta en alcanzar la mayor concordancia posible entre aquello que se dice (compromiso de la organización con sus stakeholders) y aquello que se hace (acciones que se ejecutan).

Uno de los errores conceptuales más comunes es la relación directa de reputación online con problemas relativos al SEO. El posicionamiento web como resultado de la recuperación de información acerca de la compañía se evidencia a través de las bases de datos de los grandes motores de búsqueda, por el uso de determinados algoritmos. 
Este tipo de pensamiento podría categorizarse como reduccionista, ya que aquella compañía que cuente con una mala reputación, probablemente no consiga subsanar sus problemas eliminando su enlace en las búsquedas con una serie de keywords predefinidos. Sus problemas persistirán tanto en la esfera online como en la offline - ámbito que suscita mayor preocupación-. La reputación, por tanto, no mejorará aunque la empresa lidere los primeros puestos en los buscadores.

Enrique Dans (2011) defiende, pese a la insistencia de algunos blogueros, que la reputación online no se mejora suprimiendo dichas críticas negativas que circulan en la Red, sino mejorando la gestión y la realidad de la organización. Es necesario que se aúnen los esfuerzos para potenciar la calidad del servicio, la atención al cliente o las políticas de gestión, entre otras iniciativas.

La optimización de los motores de búsqueda (Search Engine Optimization, SEO) tan sólo puede repercutir sobre la notoriedad de la compañía, no sobre el reconocimiento que los stakeholders de una compañía manifiestan en Internet sobre el comportamiento corporativo de la misma a partir del grado de cumplimiento de sus compromisos, que es la definición de reputación online que plantea Jerusalem Hernández (2011:204), a partir de la establecida por Justo Villafañe.

En definitiva, la reputación online se crea de forma distribuida, asincrónica, dinámica y permanente. Además, a partir del tono de las opiniones, se plantea una tipología de opinantes: apóstoles, neutrales y detractores (del Fresno, 2011). En relación a esta clasificación, cabe mencionar la teoría de Ángel Alloza (2010: 134) en la que expresa que las percepciones cobran significado en tres ámbitos fundamentales: lo físico (conocimiento), lo funcional (acción) y lo emocional (como su propio nombre indica, alude a las emociones). Así pues, para el profesional responsable de la reputación online será básico entender y gestionar cada uno de los puntos de contacto con sus stakeholders, tanto físicos como virtuales.

\section{Roles profesionales para la gestión de la reputación online}

El carácter transversal de la reputación se traslada a la reputación online, demandando un equipo humano que coordine las múltiples tareas mencionadas con anterioridad.

Se identifican, de esta manera, nuevas figuras en la gestión reputacional. Por una parte, el controller de intangibles, con un perfil financiero. Desarrolla el Cuadro de Mando y aquellos indicadores más sofisticados que revalorizan el peso de los intangibles. Junto a este perfil se dibuja el del Agente de Transformación o Transformacional, cuya función básica se centra en analizar la realidad y proponer estrategias para mejorarla. Por otra parte, se destaca la figura del community manager, que en la investigación descrita por Sandra Sotillo supone un reto: «en realidad se trata de incorporar a las funciones del community manager aquellas relacionadas con la reputación de la empresa en el medio online» (Sotillo, 2011: 182). 
Como se puede constatar a lo largo del proceso de investigación, el órgano de gestión reputacional offline debe coordinar, a su vez, la reputación online para actuar bajo la máxima coherencia.

Stephan Fuetterer (2010:27) destaca que el responsable debería ser la persona que asegure que los programas desarrollados a través de medios sociales se adecuan a la filosofía, misión, visión y valores de la empresa. No tiene por qué ser un especialista absoluto en asuntos de la Web 2.0, pero sí debe poseer unos conocimientos que potencien las posibilidades de trabajo con las plataformas interactivas y, a su vez, el impacto positivo sobre la organización.

En cambio, quienes demandan un conocimiento exhaustivo acerca de las plataformas y herramientas que ofrece la Web 2.0 son los profesionales que desarrollan la estrategia e implementan los programas en los medios sociales. Dichos profesionales se sitúan en el nivel inmediatamente posterior respectivo al descrito con anterioridad.

\section{Modelos de gestión de la reputación online}

Resulta necesario el desarrollo de modelos de gestión de la reputación online que adquieran una dimensión holística. Si bien es necesario obtener un análisis de imagen, notoriedad o visibilidad positiva en la red, se pretende alcanzar una rentabilidad reputacional, integrada en los principios empresariales (Hernández, 2011).

Por otro lado, Fernando Polo (2011:13) subraya la importancia de diseñar una metodología concreta para una gestión eficiente de la reputación. Además, señala que la medición es un aspecto que se debe tener en cuenta tras la actuación y no a la inversa.

Partiendo de la reputación corporativa, desde sus diferentes vertientes, Sandra Sotillo (2011) defiende que no existe un modelo ideal de gestión reputacional sino que cada empresa requiere un modelo propio. Por este motivo, la mayoría de las corporaciones se encuentra en un proceso de creación o reformulación de un modelo de gestión, debido a la dificultad que entraña.

Como se ha explicado con anterioridad, en la dimensión 2.0 al individuo le resulta más fácil alcanzar un mayor poder de influencia que en la esfera offline. En determinados instantes, puede ejercer, incluso, el rol de líder de opinión. Por ello, los mecanismos de alerta activa facilitan la detección de esta serie de cambios. Dicho fenómeno es denominado reputación distribuida, Cristina Aced y Magali Benítez han sido las precursoras en explorar el concepto.

El problema surge cuando los flujos de información no se encuentran controlados, ya que pueden originar una tormenta que repercuta sobre la reputación online de las empresas. Miguel del Fresno (2011), por su parte, expresa que la traba no es el exceso de información sino el filtro o propósito. 


\section{Principales conclusiones de la investigación}

La concepción de la reputación online como reconocimiento del comportamiento de la corporación. Refuta la hipótesis de algunos profesionales basada en que la reputación online es la disciplina que se fundamenta en la recopilación y análisis de las opiniones más notorias en Internet y, por tanto, la monitorización es el elemento decisivo.

Como se puede constatar, la evolución del concepto no muestra variaciones significativas para los expertos en la materia. Sin embargo, en el imaginario colectivo, se han revelado determinados matices que alteran la interpretación inicial del mismo. La fase centrada exclusivamente en la monitorización y el rastreo ha cedido el paso a una nueva etapa analítica, que promueve una gestión estratégica.

Por otro lado, los social media y, en concreto, las redes sociales existentes en la actualidad, tenderán a sufrir modificaciones que perpetúen su uso. El proceso comunicativo online dispone con una base de sólidos cimientos, que fortalecerá el fenómeno manteniendo la relevancia actual. En definitiva, Internet ha supuesto un avance sin precedentes en la Historia de la Comunicación y ha fomentado nuevas formas de relación entre las empresas y sus stakeholders.

Respecto a los espacios sociales, en ellos se encuentran difuminados los juicios de valor que impulsan la reputación online. Por tanto, una lectura exhaustiva de las opiniones de los grupos de interés favorecerá la extracción de insights, que más tarde se utilizarán en programas de gestión concretos al servicio de la estrategia corporativa.

En cuanto a la polarización de las opiniones en Internet, pese a las conjeturas, se concluye que el usuario no adopta una actitud más crítica en la esfera online.

La consolidación de determinados espacios web ha sosegado a los responsables de comunicación online, quienes comienzan a apostar, de forma cautelosa, por iniciar un diálogo con sus stakeholders.

Existe una ferviente demanda de equipos multidisciplinares para afrontar la gestión reputacional online. Se busca un perfil especializado en comunicación y nuevas tecnologías; otro analítico, que aporte recomendaciones estratégicas basadas en técnicas de investigación social y programas que haya implementado la corporación; y otro con un carácter económico, que sea capaz de enlazar las cuestiones estratégicas con las técnicas para generar valor a través de la reputación corporativa. Además, la gestión de la reputación online requiere transversalidad y participación interdepartamental.

Por lo que respecta al rol profesional denominado community manager, se confirma la sobrevaloración de su figura.

En el organigrama empresarial el responsable de la reputación online debe ubicarse junto a la Alta Dirección o dependiendo directamente de ésta (posición similar a la del director de comunicación). 
En las diferentes clasificaciones de herramientas de gestión y monitorización se averiguan carencias analíticas por lo que respecta a la información cualitativa. La tecnología precisa un software más avanzado, ya que, por el momento, no procesa los significados simbólicos según requiere el discurso social.

Se propone la utilización de listas blancas como principal recurso identificativo de las opiniones relevantes de los stakeholders.

Las metodologías más arraigadas engloban, primordialmente, la escucha y, por tanto, el análisis de las opiniones de los stakeholders; la participación, tras el conocimiento exhaustivo y la formación de los profesionales; y la planificación de actuaciones futuras, por tanto, el diálogo con los diferentes públicos de interés.

Respecto a los modelos de gestión más asentados, cabe destacar que comparten como ejes fundamentales la investigación y la monitorización. Sin embargo, al igual que en la reputación corporativa offline, no existe un único modelo ni un modelo ideal. Una condición esencial para la buena gestión reputacional es llevar a cabo un procedimiento coherente con la estrategia reputacional global.

Como conclusión general, cabe destacar la necesidad de participación de las corporaciones en una sociedad tecnológica que fomenta la generación de contenidos y la transmisión del conocimiento a través de la red de redes, Internet. Para empresas y grupos de interés esta nueva forma de relación supone un sinfín de oportunidades. La nueva lógica empresarial evidencia la importancia de la reputación corporativa como valor en alza. La inclusión de la Web 2.0 muestra el brillo de una cara distinta, pero perteneciente al mismo diamante (Hernández, 2011). Este resplandor no debe deslumbrar a los profesionales del sector. Por el contrario, les orientará en su camino para alcanzar los objetivos estratégicos. Contemplamos, pues, la reputación online en el marco de la reputación corporativa, como un intangible de carácter holístico e integrador.

Entre las diferentes líneas de investigación futuras que han ido surgiendo, de forma paulatina, a lo largo de la investigación, cabe destacar una concreta: el análisis de la reputación online en una selección de grandes, medianas y pequeñas empresas. Con ello, se demostrará la predisposición de algunas empresas pequeñas por la gestión reputacional en la esfera online. Por otro lado, el conocimiento de las funciones que se desempeñan en grandes empresas evidenciará su grado de implicación en el desarrollo de nuevas tendencias del corporate centradas en las nuevas tecnologías.

\section{Referencias}

Aced, Cristina et al. (2010). Visibilidad. Cómo gestionar la reputación en Internet. Barcelona: Gestión 2000. 
Alloza, Ángel (2010). Del Maquiavelo a la Neurociencia: las marcas que queremos. En: Villafañe, Justo. La comunicación empresarial y la gestión de los intangibles en España y Latinoamérica. Madrid: Pearson.

Carrera, Roberto (2008). Lo que no es y podría ser la Reputación Online. En: blog de Roberto Carreras disponible en http://robertocarreras.es. Consultado en 10/2011.

Celaya, Javier (2007). Comunicación empresarial 2.0. La función de las nuevas tecnologías sociales en la estrategia de comunicación empresarial. Madrid: Grupo BPMO.

Cerezo, Julio (2011). Presentación. En: Cuadernos de comunicación evoca. Identidad digital y reputación online, núm. 5. Madrid: Evoca Comunicación e Imagen, 3.

Cobo Romaní, Cristóbal; Pardo Kuklinski, Hugo (2007). Planeta Web 2.0 Inteligencia colectiva o medios fast food. Barcelona/México: Grup de Recerca d Interaccions Digitals-Universitat de Vic/Flacso México.

Dans, Enrique (2010). Seguimiento y evaluación: mitos y leyendas online en la nueva era digital 2.0. Video disponible en: www.reputacioncorporativa.org. Consultado en 07/ 2011.

Esquinas, David (2010). Evolución Digital. El consumidor como medio. Optimedia Intelligence. En: www.optimediaintelligence.es. Consultado en 11/ 2011.

Fresno, Miguel del (2011). Conferencia en campus party 2011. En: blog de Miguel del Fresno disponible en http://quor-wom.blogspot.com/. Consultado en 11/2011.

Fuetterer, Stephan (2010). Mi comunidad...¿Me quiere o no me quiere? Social media y Web 2.0 para directivos, comunicadores y emprendedores. BestRelations S.A. En: www.bestrelacions.com. Consultado en 10/ 2011.

Gil, Víctor; Romero, Felipe. (2008). Crossumer. Claves para entender al consumidor español de la nueva generación. Madrid: Gestión 2000.

Hannintong, Terry (2006). Cómo medir y gestionar la reputación de su empresa, Instituto de Análisis de Intangibles. Barcelona: Ediciones Deusto.

Hernández, Jerusalem (2011). Reputación online: necesidad de un marco conceptual y modelo de gestión. En Villafañe, Justo. La comunicación empresarial y la gestión de los intangibles en España y Latinoamérica. Madrid: Pearson.

Levine, Rick et al. (2000). El Manifiesto Cluetrain. Barcelona: Deusto.

Orihuela, José Luis (2011). Mundo Twitter: Una guía para comprender y dominar la plataforma que cambió la red. Barcelona: Alienta. 
Polo, Fernando (2011). La gestión de la reputación 2.0. En: Cuadernos de Comunicación EVOCA, Identidad digital y reputación online, núm. 5. Madrid: Evoca Comunicación e Imagen, 11-16.

Sotillo, Sandra (2011). Modelos y buenas prácticas en la gestión de la reputación corporativa”. En: Villafañe, Justo. La comunicación empresarial y la gestión de los intangibles en España y Latinoamérica. Madrid: Pearson.

Villafañe, Justo (2006). Quiero trabajar aquí. Seis claves de la reputación interna. Madrid: Pearson, Pretince Hall.

Weber Sandwick, (2009). Risky Business. Reputations online. Economist Intelligence Unit. En: http://www.online-reputations.com/DLS/RiskyBusiness_WhitePaper_US.pdf. Consultado en 11/ 2011.

\section{Referencia de este artículo:}

Vaquero Collado, Alicia (2012). La reputación online en el marco de la comunicación corporativa. Una visión sobre la investigación de tendencias y las perspectivas profesionales. En: adComunica. Revista Científica de Estrategias, Tendencias e Innovación en Comunicación, $\mathrm{n}^{0} 3$. Castellón: Asociación para el Desarrollo de la Comunicación adComunica, Universidad Complutense de Madrid y Universitat Jaume I, 49-63. DOI: http://dx.doi.org/10.6035/21740992.2012.3.4 